\title{
Virus spread in complete bi-partite graphs
}

\author{
J.S. Omic ${ }^{1}$, R. E. Kooij ${ }^{1,2}$ and P. Van Mieghem ${ }^{1}$ * \\ ${ }^{1}$ Faculty of Electrical Engineering, Mathematics, and Computer Science \\ Delft University of Technology, P.O. Box 5031, 2600 GA Delft \\ ${ }^{2}$ TNO Information and Communication Technology, \\ P.O. Box 5050, 2600 GB Delft, The Netherlands
}

\begin{abstract}
In this paper we study the spread of viruses on the complete bi-partite graph $K_{M, N}$. Using mean field theory we first show that the epidemic threshold for this type of graph satifies $\tau_{c}=\frac{1}{\sqrt{M N}}$, hence, confirming previous results from literature. Next, we find an expression for the average number of infected nodes in the steady state. In addition, our model is improved by the introduction of infection delay. We validate our models by means of simulations. Inspired by simulation results, we analyze the probability distribution of the number of infected nodes in the steady state for the case without infection delay. The mathematical model we obtain is able to predict the probability distribution very well, in particular, for large values of the effective spreading rate. It is also shown that the probabilistic analysis and the mean field theory predict the same average number of infected nodes in the steady state. Finally, we present a heuristic for the prediction of the extinction probability in the first phase of the infection. Simulations show that, for the case without infection delay, this time dependent heuristic is quite accurate.
\end{abstract}

\section{Keywords}

Computer Virus, Epidemiology, Modeling, Simulation

\section{INTRODUCTION}

The theory of the spectra of graphs contains many beautiful results, that relate physical properties of a network, such as for instance robustness, diameter and connectivity, to eigenvalues of matrices associated with the graph, see e.g. [2], [11]. Recently it has been shown, see [8], [4], that the spectral radius of a graph (i.e. the largest eigenvalue of its corresponding adjacency matrix) plays an important role in modeling virus propagation in networks. In fact, in [8] *Email: j.s.omic@ewi.tudelft.nl, robert.kooij@tno.nl and
p.vanmieghem@ewi.tudelft.nl

Permission to make digital or hard copies of all or part of this work for personal or classroom use is granted without fee provided that copies are not made or distributed for profit or commercial advantage and that copies bear this notice and the full citation on the first page. To copy otherwise, to republish, to post on servers or to redistribute to lists, requires prior specific permission and/or a fee. Bionetics'07, December 10-13, 2007, Budapest, Hungary

Copyright 2007 ICST 978-963-9799-11-0 . and [4] the Susceptible-Infected-Susceptible (SIS) infection model is considered. The SIS model assumes that a node in the network is in one of two states: infected and therefore infectious, or healthy and therefore susceptible to infection. The SIS model usually assumes instantaneous state transitions. Thus, as soon as a node becomes infected, it becomes infectious and likewise, as soon as a node is cured it is susceptible to re-infection. There are many models that consider more aspects like incubation periods, variable infection rate, a curing process that takes a certain amount of time and so on [3], [6], [9]. In epidemiological theory, many authors refer to an epidemic threshold $\tau_{c}$, see for instance $[3],[1],[6]$ and [10]. If it is assumed that the infection rate along each link is $\beta$ while the cure rate for each node is $\delta$ then the effective spreading rate of the virus can be defined as $\tau=\beta / \delta$. The epidemic threshold can be defined as follows: for effective spreading rates below $\tau_{c}$ the virus contamination in the network dies out - the mean epidemic lifetime is of order $\log n$, while for effective spreading rates above $\tau_{c}$ the virus is prevalent, i.e. a persisting fraction of nodes remains infected with the mean epidemic lifetime of the order $e^{n^{\alpha}}$. In the case of persistence we will refer to the prevailing state as a metastable state or steady state. It was shown in [8] and [4] that $\tau_{c}=1 / \rho(A)$ where $\rho(A)$ denotes the spectral radius of the adjacency matrix $A$ of the graph. Recently, the epidemic threshold formula has also been verified by using the $N$-intertwined model, which consists of a pair of interacting continuous Markov chains, see [12]. Although this main result of [8] is very nice, we ought to mention that it was derived under a number of simplifying conditions. For instance it was assumed that for a fixed time step the probability that a nodes gets cured after infection from neighbors is $1 / 2$. In addition, [8] does not provide an explicit expression for the fraction of infected nodes in the epidemic steady state. In this paper we circumvent these drawbacks by using an alternative approach to derive an expression for the epidemic threshold and the epidemic steady state for complete bi-partite graphs. In addition, we show that the $N$-intertwined model, introduced in [12], is analytically solvable for the complete bi-partite graph. Moreover, we show, in more detail than in [12], deviations from the $N$-interwined model for the complete bi-partite graph.

The rest of the paper is organized as follows. In Section 2 we discuss the classical model by Kephart and White which describes the spread of a virus on regular graphs. In Section 3 we derive and analyze the spread of viruses on complete bi-partite graphs. In Section 4 we take the effect of infection delay on virus spread on complete bi-partite graphs into 
account. In Section 5 we validate our results through simulation analysis. The model is reinforced with probabilistic analysis in Section 6. We summarize our results in Section 7 .

\section{VIRUS SPREAD ON REGULAR GRAPHS}

In order to explain our model for virus spread on complete bi-partite graphs, it is useful to first discuss the spread of viruses over a simpler network, i.e. the connected regular graph. This model is based on a classical result by Kephart and White [6] for SIS models.

We consider a connected graph on $N$ nodes where every node has degree $k$. We denote the number of infected nodes in the population at time $t$ by $I(t)$. If the population $N$ is sufficiently large, we can convert $I(t)$ to $i(t) \equiv I(t) / N$, a continuous quantity representing the fraction of infected nodes. Now the rate at which the fraction of infected nodes changes is due to two processes: susceptible nodes becoming infected and infected nodes being cured. Obviously, the cure rate for a fraction $i$ of infected nodes is $\delta i$. The rate at which the fraction $i$ grows is proportional the fraction of susceptible nodes, i.e. $1-i$. For every susceptible node the rate of infection is the product of the infection rate per node $(\beta)$, the degree of the node $(k)$ and the probability that on a given link the susceptible node connects to an infected node (i).

Therefore we obtain the following differential equation describing the time evolution of $i(t)$ :

$$
\frac{d i}{d t}=\beta k i(1-i)-\delta i
$$

The solution to Eq. (1) is

$$
i(t)=\frac{i_{0}(1-\rho)}{i_{0}+\left(1-\rho-i_{0}\right) e^{-(\beta k-\delta) t}},
$$

with a steady state solution

$$
i_{\infty}=1-\rho,
$$

where $\rho=\frac{\delta}{\beta k}$, and $i_{0}$ is the initial fraction of infected nodes.

Obviously an epidemic steady state only exists if $i_{\infty}>0$. Because we can rewrite Eq. (3) as

$$
i_{\infty}=\frac{\beta k-\delta}{\beta k}
$$

we can conclude that the epidemic threshold satisfies

$$
\tau=\frac{1}{k} .
$$

Because for $k$-regular graphs the spectral radius of the adjacency matrix is equal to $k$, see [2], Eq. (5) is in line with the result by [8].

\section{VIRUS SPREAD ON COMPLETE BIPAR-}

\section{TITE GRAPHS}

In this section we will consider complete bi-partite graphs. A complete bi-partite graph $K_{M, N}$ consists of two disjoint sets $S_{1}$ and $S_{2}$ containing respectively $M$ and $N$ nodes, such that all nodes in $S_{1}$ are connected to all nodes in $S_{2}$, while within each set no connections occur. Figure 1 gives an example of a complete bi-partite graph on 6 nodes.

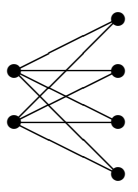

Figure 1: Complete bi-partite graph $K_{2,4}$

Notice that (core) telecommunication networks often can be modeled as a complete bi-partite topology. For instance, the so-called double-star topology (i.e. $K_{M, N}$ with $M=2$ ) is quite commonly used because it offers a high level of robustness against link failures. For example, the Amsterdam Internet Exchange (see www.ams-ix.net), one of the largest public Internet exchanges in the world, uses this topology to connect its four locations in Amsterdam to two high-density Ethernet switches. Sensor networks are also often designed as complete bi-partite graphs.

We will now derive a model for virus spreading on the complete bi-partite graph $K_{M, N}$. Without loss of generality we can assume $M \leq N$. We denote the number of infected nodes belonging to $S_{2}$ at time $t$ by $I(t)$. Again, we use the argument that for $N$ sufficiently large the continuous fraction $i(t) \equiv I(t) / N$ represents the fraction of infected nodes in $S_{2}$. The cure rate for the fraction $i$ of infected nodes in $S_{2}$ is $\delta i$. The rate at which the fraction $i$ grows is proportional the fraction of susceptible nodes $1-i$. For every susceptible node in $S_{2}$ the rate of infection is the product of the infection rate per node $(\beta)$, the degree of the node $(M)$ and the fraction of nodes in $S_{1}$ that is infected at time $t$. This latter fraction will be denoted by $j(t)$.

Therefore we obtain the following differential equation describing the time evolution of $i(t)$ :

$$
\frac{d i}{d t}=\beta M j(1-i)-\delta i .
$$

To derive the steady state of $j(t)$, which will be denoted as $j_{\infty}$, we treat the dynamics in each node of $S_{1}$ as a two-state Markov process, with a susceptible and an infectious state. Let us denote the steady state of Eq. (6) as $i_{\infty}$. Then, because each node in $S_{1}$ is connected to $N$ nodes of which a fraction $i_{\infty}$ is infectious, the rate at which a node in $S_{1}$ goes from susceptible to infectious is $\beta N i_{\infty}$. The rate at which a node in $S_{1}$ changes from infectious to susceptible is $\delta$. Therefore the steady state probability that a node in $S_{1}$ is infected satisfies:

$$
j_{\infty}=\frac{\beta N i_{\infty}}{\beta N i_{\infty}+\delta}
$$

If we substitute Eq. (7) and $i=i_{\infty}$ into Eq. (6) and solve the right hand side with respect to $i_{\infty}$ then we obtain the steady state solution for the fraction of infected nodes in $S_{2}$ : 


$$
i_{\infty}=\frac{M N \beta^{2}-\delta^{2}}{\beta N(\beta M+\delta)}
$$

Because an epidemic steady state only exists if $i_{\infty}>0$, Eq. (8) yields the epidemic threshold:

$$
\tau=\frac{1}{\sqrt{M N}} .
$$

This complies with [8] because according to [2] the spectral radius of the adjacency matrix of the graph $K_{M, N}$ is equal to $\sqrt{M N}$. Notice that for the case $M=N$ the graph $K_{M, N}$ is in fact regular and Eq. (9) reduces to Eq. (5) with $k=N$.

For effective spreading rates above the epidemic threshold the epidemic steady state $\eta_{\infty}$ for the complete bi-partite graph $K_{M, N}$ satisfies

$$
\eta_{\infty}=\frac{M j_{\infty}+N i_{\infty}}{M+N}
$$

Substitution of Eq. (8) and Eq. (7) into Eq. (10) yields

$$
\eta_{\infty}=\frac{\left(M N \beta^{2}-\delta^{2}\right)(\beta N+\beta M+2 \delta)}{\beta(M+N)(\beta M+\delta)(\beta N+\delta)} .
$$

It is easy to verify that for the case $M=N$, Eq. (11) reduces to Eq. (4), with $k=N$.

\section{THE IMPACT OF INFECTION DELAY}

So far we have assumed that once a node is infected, it instantaneously becomes infectious. In reality, there may be a time lag between the arrival of a virus at a node and the time this node itself starts to spread the virus. A virus could lie dormant on a host due to user inactivity or because the virus was designed in this manner for stealth reasons.

In [9] Wang and Wang have studied the impact of infection delay on the epidemic threshold and the epidemic steady state for regular graphs. In [9] the infection delay $\epsilon$ is defined as the length of time between the virus arrival at a node and the instant the node becomes infectious.

It is shown in [9] that the steady state for the fraction of infectious nodes satisfies

$$
i_{\infty}=\frac{\beta k-\delta e^{\delta \epsilon}}{\beta k},
$$

which yields for the epidemic threshold:

$$
\tau=\frac{e^{\delta \epsilon}}{k}
$$

Thus, the infection delay increases the epidemic threshold, which means that infection delay makes an epidemic die out more easily.
In this section we will study the impact of infection delay on virus spread on complete bi-partite graphs.

Analogous to Eq. (6) we can derive the following delaydifferential equation for the evolution of $i(t)$, which as before, denotes the fraction of infected nodes in $S_{2}$ at time $t$ :

$$
\frac{d i(t)}{d t}=\beta M j(t-\epsilon) e^{-\delta \epsilon}(1-i(t))-\delta i(t),
$$

where $j(t-\epsilon)=0$ for $t<\epsilon$ and $j(t)$ denotes the fractions of nodes in $S_{1}$ that is infectious at time $t$. For $t \geq \epsilon$, the probability that a node in $S_{1}$ is infectious is the probability that the node was already infected at time $t-\epsilon$, since all nodes infected between $t-\epsilon$ and $t$ are still being delayed. Curing a node during the infection delay period $\epsilon$ results in the $e^{-\delta \epsilon}$ factor.

Let us denote the steady state of Eq. (14) as $i_{\infty}$. We solve for $i_{\infty}$ by setting the right hand side of Eq. (14) equal to zero and $j(t-\epsilon)=j_{\infty}$. Analogous to Eq. (7) we find for $j_{\infty}$

$$
j_{\infty}=\frac{\beta N i_{\infty} e^{-\delta \epsilon}}{\beta N i_{\infty} e^{-\delta \epsilon}+\delta},
$$

where the $e^{-\delta \epsilon}$ factor corresponds with the probability that a node is cured during the infection delay period $\epsilon$.

Plugging Eq. (15) and $i=i_{\infty}$ into Eq. (14) and solving the right hand side with respect to $i_{\infty}$ we obtain the steady state solution for the fraction of infected nodes in $S_{2}$ :

$$
i_{\infty}=\frac{M N \beta^{2}-\delta^{2} e^{2 \delta \epsilon}}{\beta N\left(\beta M+\delta e^{\delta \epsilon}\right)},
$$

which yields for the epidemic threshold:

$$
\tau=\frac{e^{\delta \epsilon}}{\sqrt{M N}}
$$

Analogous to the previous section it can be shown that for effective spreading rates above the epidemic threshold the epidemic steady state $\eta_{\infty}$ for the complete bi-partite graph $B_{M, N}$ with infection delay $\epsilon$ satisfies

$$
\eta_{\infty}=\frac{\left(M N \beta^{2}-\delta^{2} e^{2 \delta \epsilon}\right)\left(\beta N+\beta M+2 \delta e^{\delta \epsilon}\right)}{\beta(M+N)\left(\beta M+\delta e^{\delta \epsilon}\right)\left(\beta N+\delta e^{\delta \epsilon}\right)} .
$$

Notice that for $\epsilon=0$ the results obtained in this section (Eqs. (16-18)) reduce to the corresponding results in Section 3 .

\section{SIMULATION ANALYSIS}




\subsection{Virus spread without infection delay}

In this section, we present a set of simulation results that will validate the mean field models proposed in the previous sections. We have conducted 500 simulations for various values of the effective spreading rate $\tau=\frac{\beta}{\delta}$ on complete bipartite graphs $K_{M, N}$ with $\{M=10, N=990\},\{M=500$, $N=500\}$. Note that for $K_{10,990}$ and $K_{500,500}$ the epidemic threshold satisfies $\tau_{c}=0.0101$ and $\tau_{c}=0.002$, respectively. The number of observed time units is 10000. Each simulation starts with 5 randomly chosen infected nodes. The virus spread is a stochastic process, and it can be expected that during evolution some of the infections die out before reaching the steady state even though the effective spreading rate is above the threshold. These evolutions have been excluded from calculations of the expected number of infected nodes in the steady state.

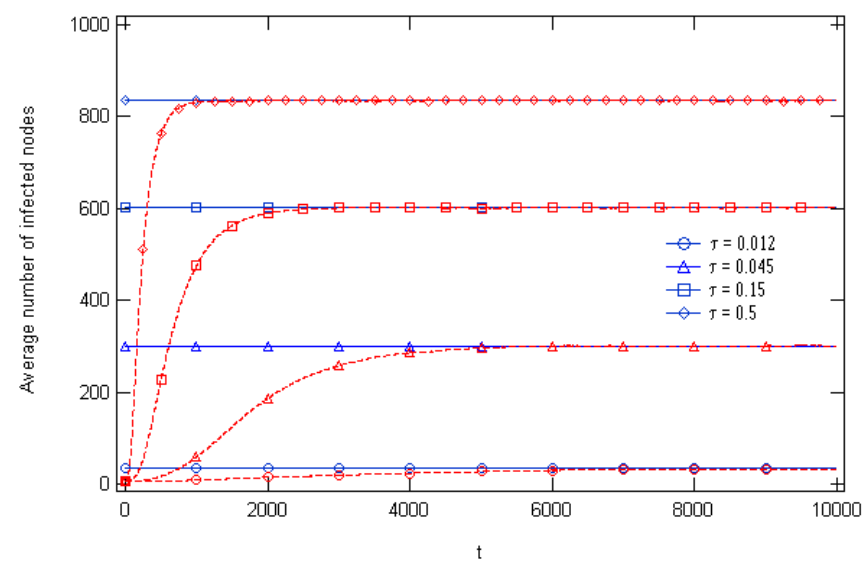

Figure 2: Average number of infected nodes for $K_{10,990}$, excluding virus epidemics that died out.

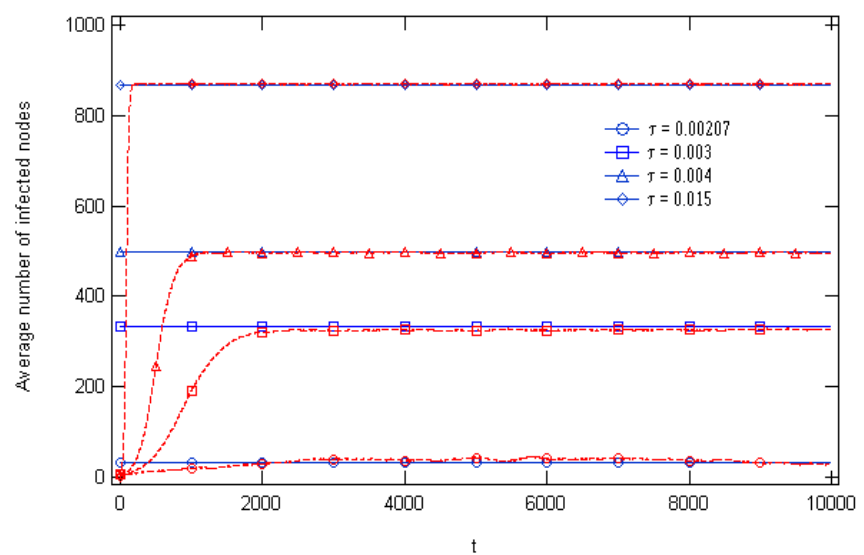

Figure 3: Average number of infected nodes for $K_{500,500}$, excluding virus epidemics that died out.

Figures 2 and 3 show the average number of infected nodes for 500 system evolutions for different values of $\tau$. The dashed lines are simulation results while full lines denote theoretical predictions. As shown, our model predicts the mean number of infected nodes in the steady state very well.

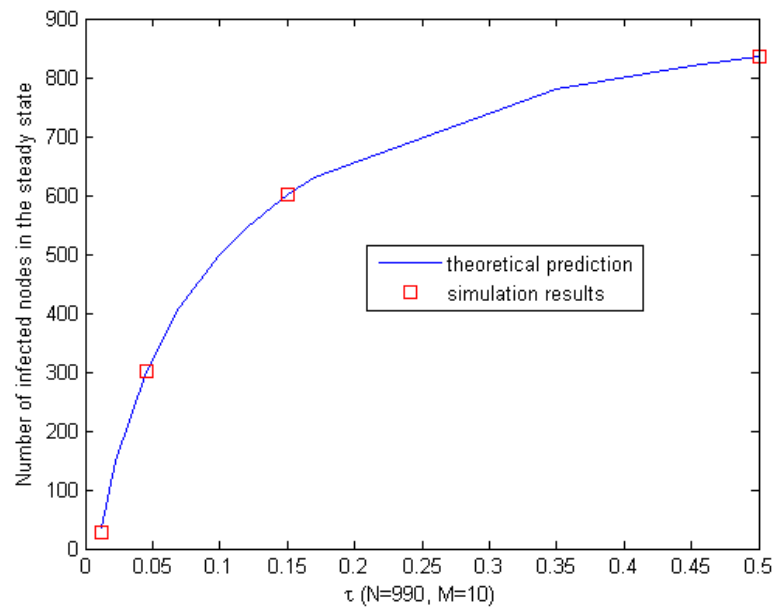

Figure 4: Number of infected nodes in the steady state for $K_{10,990}$

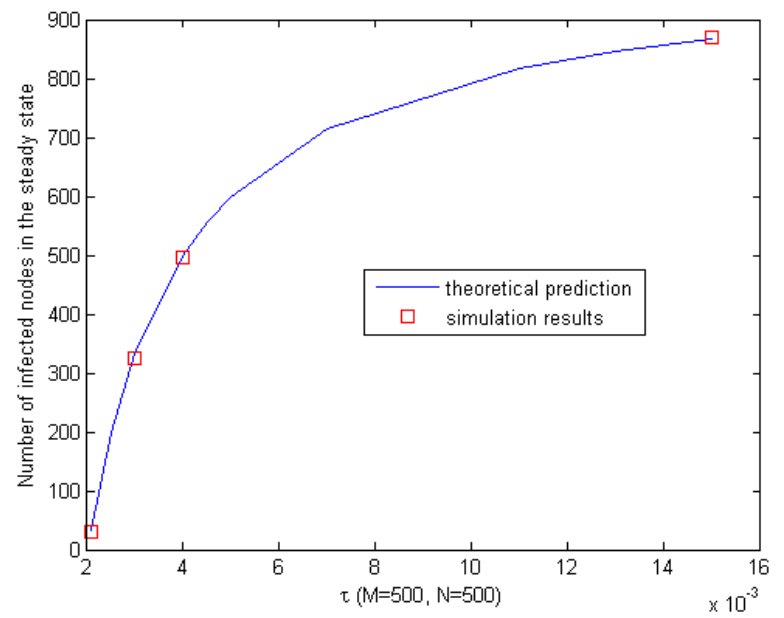

Figure 5: Number of infected nodes in the steady state for $K_{500,500}$

Figures 4 and 5 show theoretical and simulated values for the mean number of infected nodes in steady state. Again, realizations of the system in which the virus died out during evolution are excluded in calculating the average. Simulation results also showed that below the threshold the virus dies out.

Figures 6 and 7 show 500 evolutions of the number of infected nodes during 10000 time units. We can observe that the number of infected nodes fluctuate around the average steady state predicted by our model. We will quantify the spread around the average steady state by means of the standard deviation $\sigma$. Figure 8 shows the the spread around the average steady state, in terms of $\sigma$, as a function of the effective spreading rate $\tau$. Note that our model, which is based 
upon mean field theory, fails to explain the fluctations observed in Figures 6 and 7. Also it cannot explain extinction of the virus before the steady state is reached for effective spreading rates above the threshold. We will deal with these issues in subsequent sections.

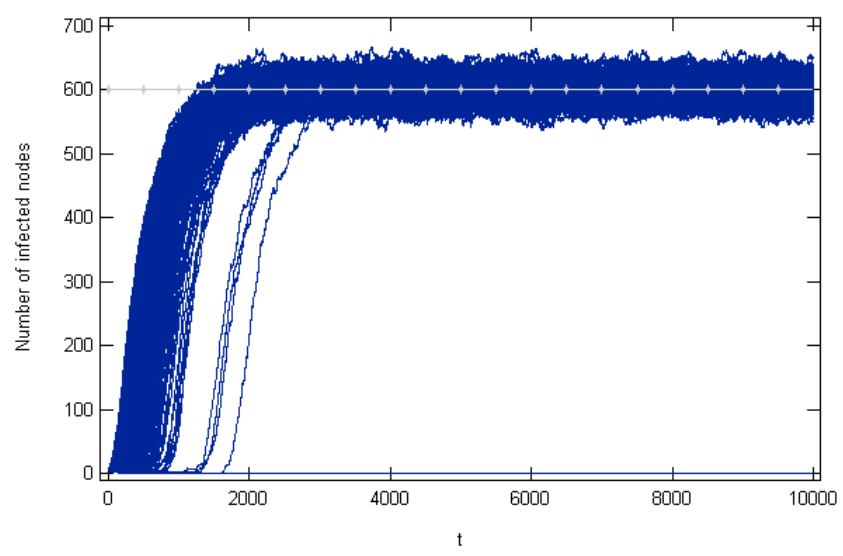

Figure 6: 500 simulations of the virus spread for $K_{10,990}, \tau=0.15$.

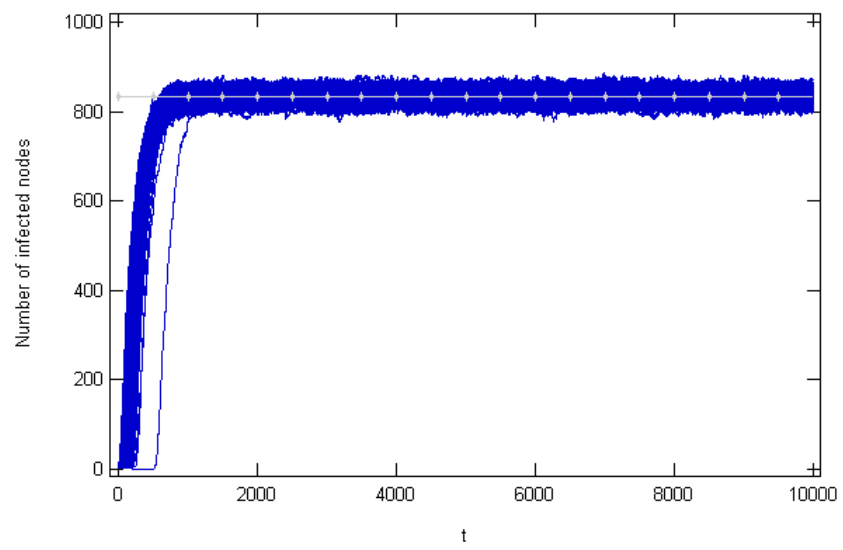

Figure 7: 500 simulations of the virus spread for $K_{10,990}, \tau=0.45$.

\subsection{The impact of infection delay}

We have conducted 500 simulations for each value of the effective spreading rate $\tau=\frac{\beta}{\delta}$ on a complete bipartite graph $K_{M, N}$ with $\{M=250, N=750\}$ and for two values of the infection delay $\varepsilon \in\{10,50\}$. The number of observed time units is 10000. Each simulation is started with 5 randomly chosen infected nodes. Again the evolutions that died out are excluded in calculating the average number of infected nodes. Figures 9 and 10, where dashed lines represent simulation results while full lines represent theoretical predictions, show that our approximation Eq. (18) predicts the steady state well for the virus spread with infecton delay.

\section{PROBABILISTIC ANALYSIS}

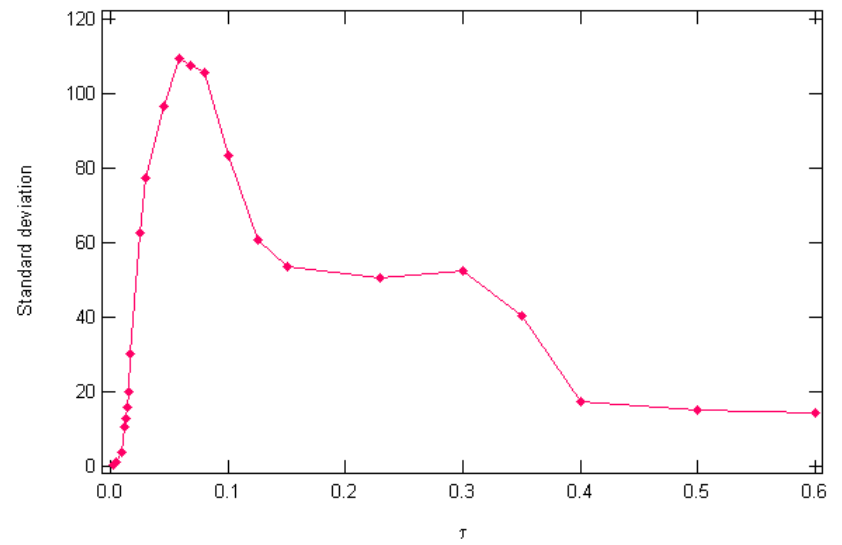

Figure 8: Spread around the steady state for $K_{10,990}$

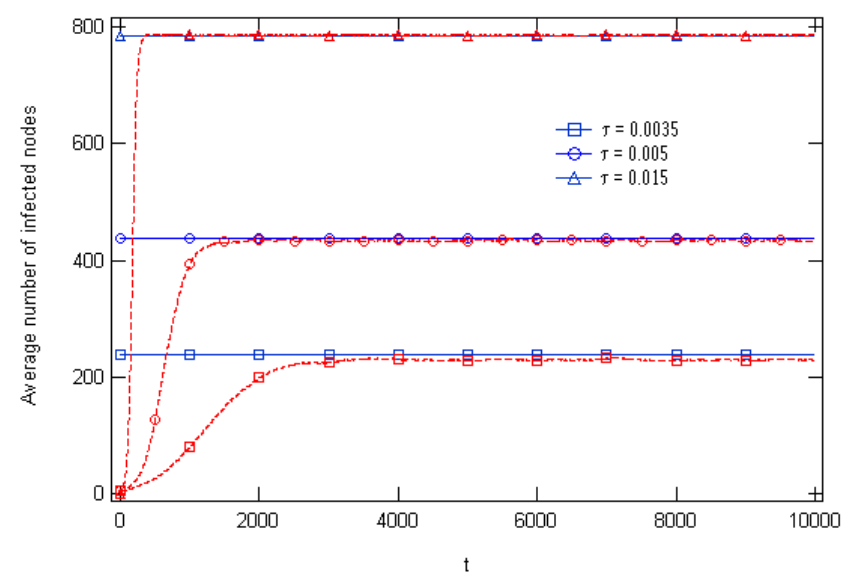

Figure 9: Average number of infected nodes for $K_{250,750}$ with infection delay $\varepsilon=10$, excluding virus epidemics that died out.

In the previous section it was shown that the mean field model has some limitations. In this section we will model the steady state of the number of infected nodes on a complete bi-partite graph $K_{M, N}$ as a statistical process using a pair of interacting continuous Markov chains. The resulting $N$ intertwined model, was introduced in [12], where it is applied to networks with any given topology.

The number of infected nodes belonging to $S_{2}$ at time $t$ is denoted by $I(t)$ and the number of infected nodes belonging to $S_{1}$ at time $t$ by $J(t)$. The probability of a $S_{2}$ node being infected is $i(t)=\frac{I(t)}{N}$ and similarly for $S_{1}$ this probability equals $j(t)=\frac{J(t)}{M}$. The arrival of infectious packets on a link and the curing process are considered to be independent Poisson processes with rates $\beta$ and $\delta$ respectively.

We will now use the interactive continuous Markov chain with two states for the nodes from $S_{1}$ and $S_{2}$, as depicted in Figure 11.

Similar work has been done on discrete time-Markov chains by Garetto et al. [5].

Every node is modeled by a continuous Markov chain with two states $X_{S_{1}}=\{0,1\}\left(X_{S_{2}}=\{0,1\}\right)$. We can now write 


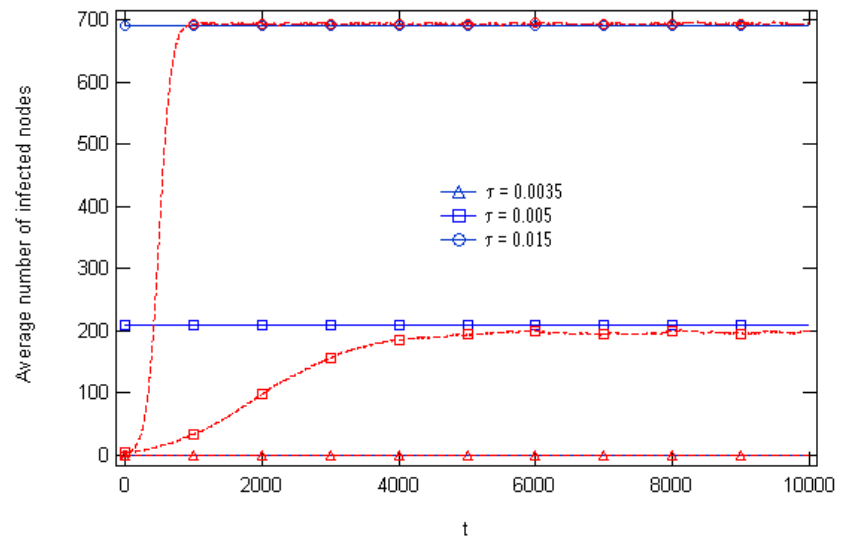

Figure 10: Average number of infected nodes for $K_{250,750}$ with infection delay $\varepsilon=50$, excluding virus epidemics that died out.
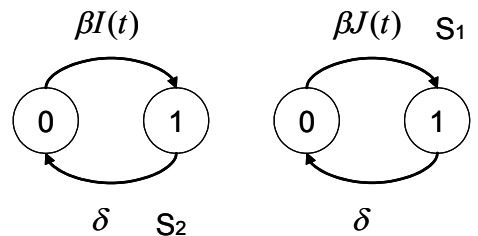

Figure 11: Markov chains for nodes of $S_{1}$ and $S_{2}$.

the infinitesimal generator $Q$, for the nodes of $S_{1}$ and $S_{2}$ respectively:

$$
\begin{aligned}
& Q_{S_{1}}(t)=\left[\begin{array}{cc}
-\beta I(t) & \beta I(t) \\
\delta & -\delta
\end{array}\right] \\
& Q_{S_{2}}(t)=\left[\begin{array}{cc}
-\beta J(t) & \beta J(t) \\
\delta & -\delta
\end{array}\right]
\end{aligned}
$$

The steady state solution satisfies, see [11]:

$$
\begin{aligned}
& Q_{S_{1}} \pi_{S_{1}}=0 \\
& Q_{S_{2}} \pi_{S_{2}}=0
\end{aligned}
$$

where the vectors $\pi_{S_{1}}$ and $\pi_{S_{2}}$ denote the steady state probabilities of a node in $S_{1}$ or $S_{2}$ being in one of two states:

$$
\begin{aligned}
& \pi_{S_{1}}=\left[\begin{array}{ll}
\operatorname{Pr}\left[X_{S_{1}}=0\right] & \operatorname{Pr}\left[X_{S_{1}}=1\right]
\end{array}\right] \\
& \pi_{S_{2}}=\left[\begin{array}{ll}
\operatorname{Pr}\left[X_{S_{2}}=0\right] & \operatorname{Pr}\left[X_{S_{2}}=1\right]
\end{array}\right]
\end{aligned}
$$

Solving this system of equations, under the condition $\operatorname{Pr}\left[X_{S_{1}}=0\right]+\operatorname{Pr}\left[X_{S_{1}}=1\right]=1$, we find:

$$
\begin{aligned}
& j_{\infty}=\operatorname{Pr}\left[X_{S_{1}}=1\right]=\frac{\tau^{2} M N-1}{\tau M(\tau N+1)}, \\
& i_{\infty}=\operatorname{Pr}\left[X_{S_{2}}=1\right]=\frac{\tau^{2} M N-1}{\tau N(\tau M+1)},
\end{aligned}
$$

We can now find the mean epidemic steady state $\eta_{\infty}$ as:

$$
\eta_{\infty}=\frac{M j_{\infty}+N i_{\infty}}{M+N}
$$

Substituting Eq. (19) in Eq. (20) yields:

$$
\eta_{\infty}=\frac{\left(M N \tau^{2}-1\right)((M+N) \tau+2)}{(M+N) \tau(M \tau+1)(N \tau+1)}
$$

This complies with Eq. (11).

The epidemic spreading is a stochastic process, and in the steady state, the system is taking a set of values around the mean epidemic steady state $\eta_{\infty}$, see also Figures 3-2. Because the steady state probability of a node being infected does not depend on other nodes the steady state probability $\operatorname{Pr}[I, J]$ satisfies:

$\operatorname{Pr}[I=x, J=y]=\left(\begin{array}{c}N \\ x\end{array}\right) i_{\infty}^{x}\left(1-i_{\infty}\right)^{N-x}\left(\begin{array}{c}M \\ y\end{array}\right) j_{\infty}^{y}\left(1-j_{\infty}\right)^{M-y}$

\subsection{Simulation results for steady state proba- bility distribution}

We conducted simulations for the complete bi-partite graph $K_{M, N}$ with $M=10, N=990$ with the effective spreading rate $\tau$ satisfying $\tau \in\{0.045,0.15,0.5\}$. Note that the epidemic threshold for this case satisfies $\tau_{c}=0.0101$. We have assumed that the system is in steady state from $t=6000$ onwards, see Figure 2. We will now compare the probability distribution for the number of infected nodes in steady state with the probability distribution given by Eq. (22). In Figure 12 dashed lines represent simulation results, full lines represent theoretical predictions. Figure 12 also contains the probabilities that the virus dies out during system evolution.

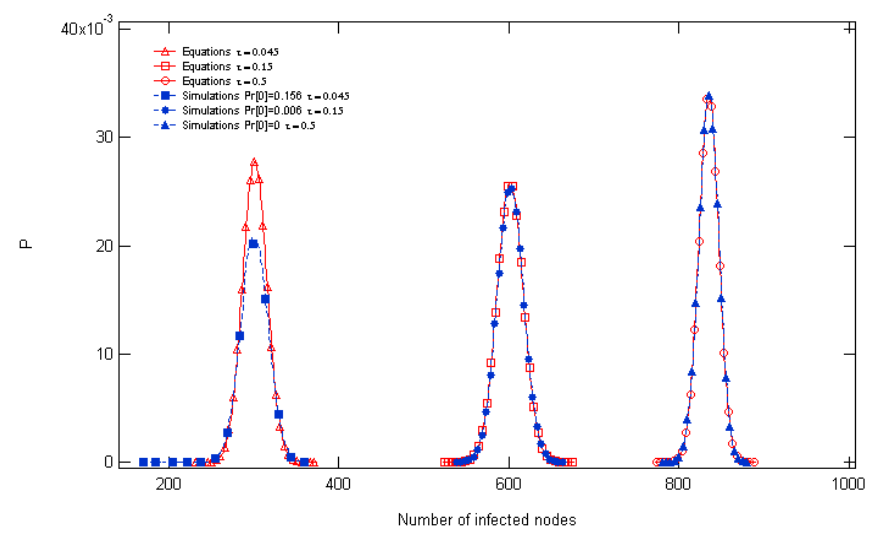

Figure 12: Probability distribution of the number of infected nodes in the steady state for $K_{10,990}$

We conclude from the simulation that Eq. (22) predicts the probability distribution of the number of infected nodes in steady state very well for large values of the effective spreading rate $\tau$. For values of $\tau$ just over the threshold our model is less accurate in predicting the probability distribution. This confirms the statement made in [12] that the $N$-intertwined model exhibits the largest deviation around $\tau=\tau_{c}$. 


\subsection{Extinction probability}

In this section we estimate the probability $p_{\text {ext }}$ that the virus dies out before it reaches the steady state. Note that, eventually, every epidemic on a finite population will die out. However, for effective spreading rates above the epidemic threshold, this will take an extremly long time in general, see also [4].

We approximate $p_{\text {ext }}$ by the probability that all initially infected nodes are cured before they infect any other node. We initially infect $N_{0}$ nodes in the larger group of nodes $S_{2}$ (consisting of $N$ nodes). Then $p_{\text {ext }}$ equals the probability that all $N_{0}$ nodes are cured before they infect any of the susceptible $M$ nodes to which they are attached, see Figure 13, where full and open circles denote infected and susceptible nodes, respectively.

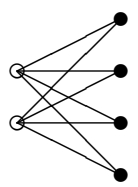

Figure 13: Complete bi-partite graph $K_{M, N_{0}}$, with $N_{0}$ infected nodes

Let us first determine the probability $p_{M}$ that one specific node will be cured before it has infected any of the susceptible $M$ nodes, before time $T$. It is assumed that the infection process (over a link) and the node curing process are independent Poisson processes with rates $\beta$ and $\delta$, respectively. Furthermore, let $T_{\beta}$ be a stochast that denotes the time it takes for a susceptible node to become infected over a link and $T_{\delta}$ denotes the time it takes for a node to cure. For the latter stochast, let $f_{T_{\delta}}(x)$ denote its corresponding probability density function. Suppose the infected node is cured at time $x$, with $0 \leq x \leq T$. This implies, that for all $M$ susceptible nodes attached to the infected node, we require $T_{\beta}>x$. Applying the law of total probability we obtain:

$$
\begin{aligned}
p_{M} & =\int_{0}^{T}\left[\operatorname{Pr}\left[T_{\beta}>x \mid T_{\delta}=x\right]\right]^{M} f_{T_{\delta}}(x) d x \\
& =\int_{0}^{T}\left(e^{-\beta x}\right)^{M} \delta e^{-\delta x} d x \\
& =\frac{\delta}{\delta+M \beta}\left(1-e^{-(\delta+M \beta) T}\right) .
\end{aligned}
$$

Because the curing processes of the $N_{0}$ infected nodes are independent, in order to obtain $p_{\text {ext }}$, we have to multiply the probabilities of each of them being cured before they infect other nodes, which leads to:

$$
p_{\text {ext }}=\left(\frac{\delta}{\delta+M \beta}\left(1-e^{-(\delta+M \beta) T}\right)\right)^{N_{0}}
$$

In order to estimate how well Eq. (23) predicts extinction of a virus spread in the first phase, we have conducted 500 simulations on the complete bi-partite graph $K_{M, N}$ with parameters $\{M=10, N=990, \tau=0.045\}$. Figure 14 shows the probability of extinction evolving in time for the case of three initially infected nodes $\left(N_{0}=3\right)$. We conclude that the simulations match the theoretical predictions quite well.

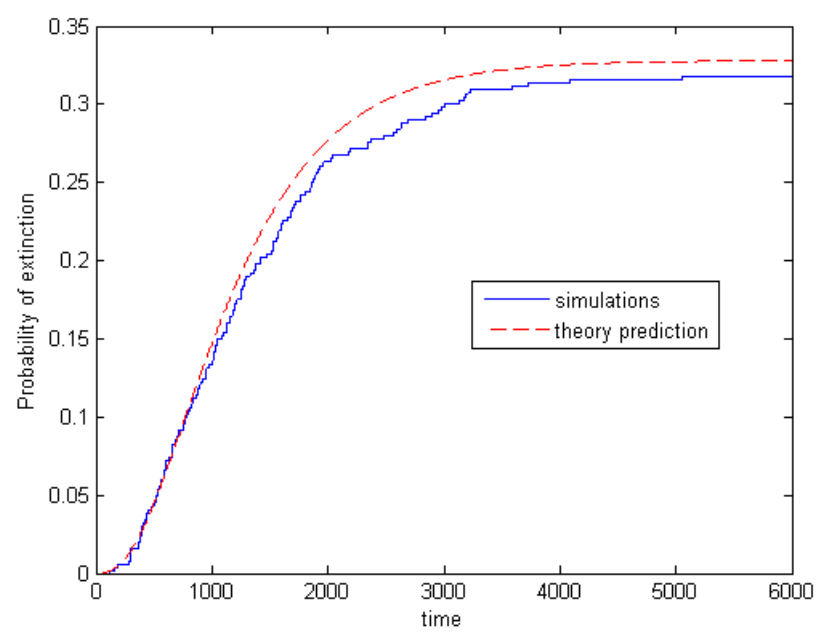

Figure 14: Extinction of the virus as a function of time for $K_{10,990}$ with $\tau=0.045$ for 3 initially infected nodes.

Figure 15 depicts $p_{\text {ext }}$ for $T=6000$ units, where the number of initially infected nodes varies between 1 and 8 .

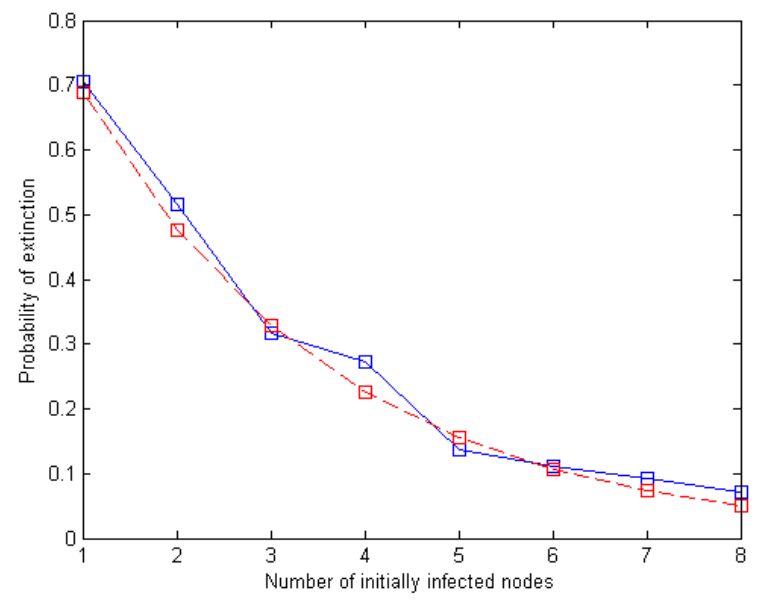

Figure 15: Extinction of the virus after $T=6000$ as a function of number of initially infected nodes, for $K_{10,990}$ with $\tau=0.045$.

\section{CONCLUSION}

In this paper we have studied the spread of viruses on the complete bi-partite graph $K_{M, N}$. Using elements of mean field theory and Markov chains we have calculated the average number of infected nodes in the steady state (Eq. (11)) and confirmed these results by means of simulations. We have also confirmed previous results of [4] and [8] about the relation between the epidemic threshold and the largest eigenvalue of the adjacency matrix of the graph over which the virus is spreading. In addition the model was improved 
by introduction of infection delay. Inspired by simulation results we have analyzed the probability distribution of the number of infected nodes in the steady state for the case without infection delay. For the complete bi-partite graph $K_{M, N}$, our mathematical model (Eq. (22)) is able to predict the probability distribution very well, in particular for large values of the effective spreading rate. It was also shown that the probabilistic analysis and the mean field theory predict the same average number of infected nodes in the steady state, see Eq. (21). Additionally we have presented a heuristic for the prediction of the extinction probability in the first phase of the infection. Simulations show that for the case without infection delay this time dependent heuristic is quite accurate.

\section{ACKNOWLEDGEMENT}

This research was supported by the Netherlands Organization for Scientific Research (NWO) under project number 643.000 .503 , and by the Next Generation Infrastructures programme (www.nginfra.nl), which is partially funded by the Dutch government.

\section{REFERENCES}

[1] N. T. J. Bailey, The Mathematical Theory of Infectious Diseases and its Applications, Charlin Griffin \& Company, London, 2nd ed., 1975.

[2] D.M. Cvetkovic, M. Doob, H. Sachs, Spectra of graphs, Theory and Applications. Johan Ambrosius Barth Verlag, Heidelberg, third edition, 1995.

[3] D.J. Daley, J. Gani, Epidemic modelling: An Introduction, Cambridge University Press, 1999.

[4] A. Ganesh, L. Massoulié and D. Towsley, The Effect of Network Topology on the Spread of Epidemics, IEEE INFOCOM2005.

[5] M. Garetto, W. Gong, D. Towsley, Modeling Malware Spreading Dynamics, IEEE INFOCOM'03, San Francisco, CA, April 2003.

[6] J.O. Kephart, S.R. White, Direct-graph epidemiological models of computer viruses, In Proceedings of the 1991 IEEE Computer Society Symposium on Research in Security and Privacy, pp. 343-359, May 1991.

[7] A. Shwartz, A. Weiss, Large Deviations for Performance Analysis, Chapmann \& Hall, London, 1995.

[8] Y. Wang, D. Chakrabarti, C. Wang, C. Faloutsos, Epidemic spreading in real networks: An eigenvalue viewpoint, 22nd Symposium in Reliable Distributed Computing, Florence Italy, Oct. 6-8, 2003.

[9] Y. Wang, C. Wang, Modeling the Effects of Timing Parameters on Virus Propagation. ACM Workshop on Rapid Malcode, Washington, DC, Oct. 27, 2003.

[10] R. Pastor-Satorras and A. Vespignani, Epidemic Spreading in Scale-Free Networks, Physical Review Letters, Vol. 86, No. 14, April, 3200-3203.

[11] P. Van Mieghem, Performance Analysis of Communication Systems and Networks, Cambridge University Press, 2006.
[12] P. Van Mieghem, J.S. Omic and R.E. Kooij, Virus Spread in Networks, submitted to IEEE Transactions on Networking. 